\title{
A prostate cancer risk calculator (PCRC-MRI): Use of clinical and magnetic resonance imaging data to predict biopsy outcome in North American men
}

Adam Kinnaird, MD; $\mathrm{PhD}^{1,2}$; Wayne Brisbane, $\mathrm{MD}^{1}$; Lorna Kwan, $\mathrm{MPH}^{1}$, Alan Priester, $\mathrm{PhD}^{3}$; Ryan Chuang, MD, MPhil ${ }^{1}$; Danielle E. Barsa, BSc${ }^{1}$; Merdie Delfin, MSN, NP${ }^{1}$; Anthony Sisk, $\mathrm{MD}^{4}$; Daniel Margolis, MD ${ }^{5}$; Ely Felker, $\mathrm{MD}^{6}$; Jim Hu, MD, MPH ${ }^{7}$; Leonard S. Marks, MD ${ }^{1}$ Department of Urology, David Geffen School of Medicine, UCLA, Los Angeles, CA, United States; ${ }^{2}$ Division of Urology, Department of Surgery, University of Alberta, Edmonton, AB, Canada; ${ }^{3}$ Department of Bioengineering, UCLA, Los Angeles, CA, United States; ${ }^{4}$ Department of Pathology \& Laboratory Medicine, UCLA, Los Angeles, CA, United States; ${ }^{5}$ Department of Radiology, Weill Cornell Medical College, New York, NY, United States; ${ }^{6}$ Department of Radiological Sciences, UCLA, Los Angeles, CA, United States; ${ }^{7}$ Department of Urology, Weill Cornell Medical College, New York, NY, United States

Funding: R01CA218547 and R01CA195505 from the National Cancer Institute and UL1TR000124 from University of California, Los Angeles Clinical and Translational Science Institute.

Cite as: Kinnaird A, Brisbane W, Kwan, et al. A prostate cancer risk calculator (PCRC-MRI): Use of clinical and magnetic resonance imaging data to predict biopsy outcome in North American men. Can Urol Assoc J 2021 October 18; Epub ahead of print.

http://dx.doi.org/10.5489/cuaj.7380

Published online October 18, 2021

Corresponding author: Dr. Leonard S. Marks, Department of Urology, David Geffen School of Medicine, UCLA, Los Angeles, CA, United States; lmarks@mednet.ucla.edu

$* * *$

\section{Abstract}

Introduction: A functional tool to optimize patient selection for magnetic resonance imaging (MRI)-guided prostate biopsy (MRGB) is an unmet clinical need. We sought to develop a prostate cancer risk calculator (PCRC-MRI) that combines MRI and clinical characteristics to aid decision-making for MRGB in North American men.

Methods: Two prospective registries containing 2354 consecutive men undergoing MRGB (September 2009 to April 2019) were analyzed. Patients were randomized into five groups, with one group randomly assigned to be the validation cohort against the other four groups as the discovery cohort. The primary outcome was detection of clinically significant prostate cancer (csPCa) defined as Gleason grade group $\geq 2$. Variables included age, ethnicity, digital rectal exam (DRE), prior biopsy, prostate-specific antigen (PSA), prostate volume, PSA density, and MRI score. Odds ratios were calculated from multivariate logistic regression comparing two models: 
one with clinical variables only (clinical) against a second combining clinical variables with MRI data (clinical+MRI).

Results: csPCa was present in 942 (40\%) of the 2354 men available for study. The positive and negative predictive values for csPCa in the clinical+MRI model were $57 \%$ and $89 \%$, respectively. The area under the curve of the clinical+MRI model was superior to the clinical model in discovery $(0.843$ vs. $0.707, \mathrm{p}<0.0001)$ and validation $(0.888$ vs. $0.757, \mathrm{p}<0.0001)$ cohorts. Use of PCRC-MRI would have avoided approximately 16 unnecessary biopsies in every 100 men. Of all variables examined, Asian ethnicity was the most protective factor (odds ratio [OR] 0.46 [0.29-0.75]) while MRI score 5 indicated greatest risk (OR15.8 [10.5-23.9]).

Conclusions: A risk calculator (PCRC-MRI), based on a large North American cohort, is shown to improve patient selection for MRGB, especially in preventing unnecessary biopsies. This tool

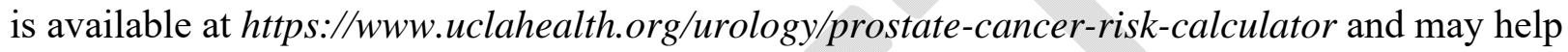
rationalize biopsy decision-making.

\section{Introduction}

Prostate cancer diagnostics have evolved in the last decade due to the addition of multiparametric magnetic resonance imaging (MRI) to guide biopsy. ${ }^{1}$ Prostate MRI allows visualization of most clinically significant prostate cancers (csPCa) and permits targeted biopsy of the lesion. ${ }^{2-4}$ The American Urological Association and the Society of Abdominal Radiology released a joint recommendation that MRI-guided biopsy (MRGB) should be performed in all men undergoing prostate biopsy when quality MRI imaging is available. ${ }^{5}$ Consequently, use of MRGB is growing. ${ }^{6}$ Because MRGB is resource-intensive, there is a need to improve selection of patients for whom the new technology would best be applied. Thus, an accessible tool that incorporates MRI data into prostate cancer risk stratification would fill an important unmet need.

Improving efficiency of prostate biopsy is also important because of the potential complications of the procedure. ${ }^{7}$ As many as $6.9 \%$ of men may be hospitalized within 30 days after prostate biopsy, most often for bacterial infection. ${ }^{7}$ Infectious complications are increasing and expensive, costing a median of over $\$ 4000$ U.S. for each event. ${ }^{8}$ In fact, approximately $50 \%$ of the costs attributed to a prostate biopsy are due to complications. ${ }^{9}$ Transperineal biopsy, which has not yet been widely adopted in the U.S. may reduce infectious complications, but pain, urinary retention, hematospermia, and hematuria remain common. ${ }^{8}$ Thus, increasing efficiency of biopsy would ease a current burden on healthcare systems.

Risk calculators are tools that use available data to predict the likelihood of an outcome and assist informed decision-making. Regarding prostate cancer, several 'pre-MRI era' risk calculators that assist with the decision to biopsy have been published. These include the Prostate Cancer Prevention Trial Risk Calculator (PCPTRC) and the European Randomized Study of Screening for Prostate Cancer (ERSPC) risk calculator. ${ }^{10,11}$ While the ERSPC risk calculator has 
been updated to include MRI data, ${ }^{12}$ currently lacking is an interactive utility for predicting biopsy outcomes based on a North American cohort that includes MRI data. This distinction in data source is a pitfall of risk calculators because it limits their use outside of the population from which they are derived. ${ }^{13}$ Therefore, we sought to create a risk calculator to predict clinically significant prostate cancer (csPCa) in men undergoing MRGB in a large North American cohort.

\section{Methods}

\section{Study design}

We analyzed data from prospective observational cohorts of men undergoing MRGB at UCLA and Cornell between 9/2009 and 4/2019 (Figure 1). These sites were selected because of lengthy commonality of personnel and technology for MRGB (>10 years). Men previously diagnosed with prostate cancer (active surveillance) were excluded. Subjects were randomized equally into 5 groups. We randomly chose 1 group $(n=469)$ to be the validation cohort against the other 4 groups $(\mathrm{n}=1885)$ as the discovery cohort.

\section{Primary outcome: Detection of csPCa defined as Gleason grade group $\geq 2$}

\section{Imaging}

Multiparametric MRI of the prostate was performed with 3 Tesla magnets with pelvic phasedarray coils. MRIs were interpreted by experienced genitourinary radiologists. Before the Prostate Imaging Reporting and Data System (PI-RADS) was introduced, regions of interest (ROI) were graded using an in-house Likert scale with scores concordant to those used in PI-RADS, version 2 (v2). ${ }^{14}$ Beginning in late 2014, ROIs were graded using the PI-RADSv2 scoring system. The ROIs were interpreted and contoured by radiologists using DynaCAD (Invivo/Philips, USA) and ProFuse software (Eigen, USA).

\section{MRI-guided biopsy}

Combined targeted and systematic biopsies detect more csPCa than either strategy alone. ${ }^{15,16}$ Therefore, all men underwent combined targeted and systematic sampling. The Artemis (Eigen, USA) MRI/US fusion device was used as previously described (Figure 2). ${ }^{15}$

\section{Statistical analyses}

T-tests (or Wilcoxon rank-sum) and Chi-square tests were used to compare patient and clinical characteristics between discovery and validation cohorts. We conducted multivariate logistic regressions on the discovery and validation cohorts to calculate odds ratios for covariates and subsequent predicted probabilities for each patient. We chose two sets of covariates a priori based on risk factors identified in other models ${ }^{12,17}$ : the clinical covariates were age, ethnicity, abnormal digital rectal exam (DRE), prior negative biopsy and PSA, and the MRI covariates were prostate volume, PSA density $(<0.15 \mathrm{vs} \geq 0.15 \mathrm{ng} / \mathrm{mL} / \mathrm{cc})$ and MRI score. Family history of 
first-degree relative with prostate cancer was included in a post-hoc analysis as an exploratory clinical variable. We constructed a 'Clinical' model that included only the clinical covariates and a 'Clinical+MRI' model that included both sets of covariates. Models using MRI score only, and MRI score in addition to PSA density were also constructed. A two-sided p-value 0.05 was considered significant. Receiver operating characteristic curves were plotted to evaluate the value of the final models as well as components of each model. We also calculated net benefit (using true positive and false positive rates) and net reduction in 'unnecessary' biopsies for both models. ${ }^{18}$ All analyses were conducted in SAS 9.4 (Cary, NC) by co-author L.K..

\section{Ethical approval}

UCLA and Cornell human research ethics boards approved of the collection and usage of patient data (UCLA: \#11001580; Cornell: \#1509016548)

\section{Results}

MRGB was performed in 2354 consecutive men (1594 from UCLA, 760 from Cornell). Mean (SD) age was 65 years (7.8) and 63\% were Caucasian. Median (IQR) PSA and prostate volume were $6.5 \mathrm{ng} / \mathrm{mL}(4.7,9.5)$ and $48 \mathrm{cc}(35,68)$, respectively. Twenty-one percent of men had a family history of prostate cancer, $38 \%$ previously had a negative biopsy and $81 \%$ had an MRI score $\geq 3$ (Table 1). At MRGB, 40\% of men had benign histology, 20\% had Gleason Grade Group 1 and 40\% had csPCa (Figure 1). There were no significant differences between men randomized to the discovery and validation cohorts used for analysis (Supplementary Table 1).

The odds ratios from the Clinical and Clinical+MRI multivariate logistic regressions for detecting csPCa in the discovery cohort are shown in Table 2 (univariate logistic regressions are shown in Supplementary Table 2). The two covariates that were associated with the greatest reduction in csPCa risk were clinical covariates Asian ethnicity ( $\mathrm{OR}=0.46,95 \% \mathrm{CI}$ 0.29-0.75) and previous negative biopsy ( $\mathrm{OR}=0.54,95 \% \mathrm{CI} 0.42-0.69)$, while the covariate associated with the greatest increase in risk was the MRI score (score 4: OR=3.96, 95\%CI 2.79-5.62; score 5: $\mathrm{OR}=15.8,95 \% \mathrm{CI}$ 10.5-23.9; referent is MRI score $<3$ ). Age, abnormal DRE, PSA, and elevated PSA density were also independently associated with increased risk of csPCa (Table 2). A posthoc analysis including available family history data $(\mathrm{n}=1552)$ in the Clinical+MRI model gives an insignificant odds ratio of 1.07 (95\% CI 0.77-1.47, $\mathrm{p}=0.72)$.

The AUC of the Clinical+MRI model was superior to that of the Clinical model in both discovery ( 0.843 vs. $0.707 ; \mathrm{p}<0.0001)$ and validation $(0.888$ vs. $0.757 ; \mathrm{p}<0.0001)$ cohorts (Figure 3$)$. The AUC of the Clinical+MRI model outperformed MRI+PSA density ( 0.843 vs. $0.796 ; p<0.0001)$ and MRI alone (0.843 vs. $0.760 ; p<0.0001)$ (Figure 3$)$.

To estimate the value of PCRC-MRI, the concept of a risk threshold was employed. Risk threshold is the actionable level of risk at which biopsy would be recommended. At a risk threshold of $20 \%$ (i.e. 0.20 probability of csPCa detection), the Clinical+MRI model reduced the number of false positives by $37 \%$ and improved the positive predictive value by $14 \%$ compared to the Clinical model (Supplementary Table 3). Furthermore, using a 20\% biopsy threshold we 
calculated a net benefit of $23.7 \%$ from the Clinical model and $27.6 \%$ from the Clinical $+\mathrm{MRI}$ model. The additional 3.9\% net benefit conferred a net reduction of 16 unnecessary biopsies in every 100 men by using the PCRC-MRI.

\section{Discussion}

Data from 2354 North American men undergoing MRGB were used to create a predictive tool for prostate cancer, the PCRC-MRI risk calculator. This instrument uses conventional clinical data and data derived from prostate MRI to determine the probability of detecting csPCa at biopsy. With a large multi-institutional sample of patients and an AUC of 0.89 in the validation cohort, the PCRC-MRI risk calculator compares favorably to other risk-assessment tools for predicting presence of csPCa. ${ }^{12,17,19}$

Approximately one million prostate biopsies are performed annually in the U.S. and 191,930 new prostate cancer cases are anticipated in $2020 .^{7,20}$ The majority of these biopsies are currently performed using ultrasound-guided sampling of the organ, an approach that dates from the 1980s. However, ultrasound fails to visualize most prostate cancers, leaving detection to a chance encounter of the biopsy needle with cancerous tissue. This 'blind' technique appears to detect fewer clinically significant cancers and more clinically insignificant tumors compared to MRGB. ${ }^{3}$ Usage of MRGB is increasing, ${ }^{6}$ but nevertheless prostate biopsy is not without complication. Nearly $7 \%$ of men may be hospitalized within one month of biopsy, most often for bacterial infection. ${ }^{7}$ Other complications are less severe, but still bothersome and may require additional medical evaluation. Use of the PCRC-MRI may help patients avoid unnecessary biopsy, mitigating detection of clinically insignificant disease and procedural complications.

Accuracy of prostate biopsy for csPCa is increased when MRI guidance is used. ${ }^{2,3,15}$ After a decade of study and hundreds of supportive conclusions, MRGB is now recommended for all men undergoing prostate biopsy if the resources permit. ${ }^{5}$ csPCa is most likely to be detected if MRGB combines targeted and systematic biopsies. ${ }^{15,16}$ The combination is required because as many as $15-25 \%$ of csPCa are invisible on MRI, necessitating the addition of systematic cores. ${ }^{16,21}$ This strategy accurately predicts final tumor grade in $96.5 \%$ of radical prostatectomy specimens. ${ }^{16}$ All patients in the cohort used to create PCRC-MRI underwent combined targeted and systematic template biopsy (Figure 2).

The PI-RADS scoring system is a universally accepted method for determining suspicion of prostate cancer on MRI. Using template mapping biopsy as 'ground truth,' a score of 5 on the updated version of PI-RADS (version 2) has high sensitivity and specificity for the presence of csPCa. ${ }^{22}$ However, mid-range scores are only moderately predictive of csPCa, and there is debate as to whether PI-RADS 3 lesions even require biopsy. ${ }^{2,15}$ One strategy that has been commonly adopted to aid in biopsy decision-making is the combination of PI-RADS score with PSA density. For example, the likelihood of detecting csPCa is elevated in a man with a PI-RADS 3 lesion and a PSA density $>0.15 \mathrm{ng} / \mathrm{mL} / \mathrm{cc}$ compared to a PI-RADS 3 lesion and a low PSA density. $^{23}$ 
PCRC-MRI, which combines multiple variables of clinical and MRI data, may especially help biopsy decision-making in a man with a midrange PI-RADS score. PCRC-MRI outperforms the commonly employed strategies of decision-making discussed above (Figure 3). Therefore, this tool may improve patient selection for MRGB and reduce the number of unnecessary biopsies. For example, two men --- both 64 years-old with PSA $6 \mathrm{ng} / \mathrm{ml}$, normal DRE, and PIRADS 3 lesions in prostates of $45 \mathrm{cc}$ volume --- may be advised to undergo biopsy. However, using PCRC-MRI, the first man (Caucasian with no previous negative biopsy) is predicted to have a $21 \%$ risk for csPCa (Supplementary Figure 1), while the second man (Asian with a previous negative biopsy) has a predicted risk of $6 \%$. If using only MRI score to determine need for biopsy, the risk for each man is approximately $25 \%$ and biopsy would be performed in both. ${ }^{4}$ However, at a risk threshold of 10,15 , or $20 \%$, a biopsy would be performed on the first man and not recommended for the second.

While other risk calculators have been proposed, none have been developed as an interactive tool using MRI data for men in North America. This is important because prostate cancer risk is influenced by variables such as ethnicity and geography. A strength of PCRC-MRI is the inclusion of ethnicity as a variable. It is of interest to note that African American ethnicity did not increase risk of csPCa. Access to care rather than biology may account for putative aggressiveness of $\mathrm{PCa}$ in African American men. ${ }^{24}$ Specifically, when MRI-guided biopsy is used there is no appreciable difference in detection rate or aggressiveness of csPCa between African American and other men. ${ }^{25}$ Asian ethnicity was the single most important protective factor indicating absence of csPCa, in accordance with recently published work. ${ }^{26}$ Other available tools either do not report ethnicity or include relatively few absolute numbers of minority men. ${ }^{12,17}$

The interaction between ethnicity and geography, influenced by environmental factors such as diet, may affect prostate cancer biology. For example, Japanese men living in Japan have markedly different gene expression in radical prostatectomy specimens compared to Japanese men living in the United States. ${ }^{27}$ Furthermore, the age-standardized rate for prostate cancer is greater in North America than Europe (73.7 vs. 62.1 per 100,000 people) ${ }^{28}$ In fact, when the PCPTRC (U.S. men) was used to predict prostate cancer in Europeans, the benefit of that risk calculator was lost. ${ }^{29}$ While the updated ERSPC risk calculator was externally validated in another set of western European men, its value has not been established in a North American population..$^{30}$ Taken together, these data help to explain the limitations of a risk calculator used outside of the population from which it is derived.

The risk threshold at which to perform biopsy will likely be the result of an informeddecision discussion between doctor and patient. In this regard, the PCRC-MRI provides information beyond that of clinical data alone. Specifically, the risk calculator improved PPV and NPV at any biopsy risk threshold above 10\% (Supplementary Table 3). At a biopsy threshold of $20 \%$, use of the PCRC-MRI would avoid 16 unnecessary biopsies in every 100 men. 
Importantly it accomplishes this refinement at no additional cost to the healthcare system, as all the input data required are standard-of-care for MRGB.

Limitations of the study include that all biopsies were performed using a transrectal approach via the same fusion device (Artemis). However, published results with other fusion devices and other biopsy approaches (transperineal) are similar to those reported here, suggesting that the biopsy platform should not materially affect the predictive utility of the instrument. The risk calculator only applies to North American men. While somewhat under-represented, African American men are not at any greater risk for PCa than Caucasian men, as discussed above. Men of Asian descent, who comprise the most rapidly growing ethnicity in North America, were wellrepresented. All MRI studies and MRGBs were performed by highly experienced radiologists and urologists, which may limit generalizability of the results. Family history of prostate cancer data were not available from one site. Nevertheless, the large sample size and prospective, uniform data collection offset the above limitations. Future modifications of the PCRC-MRI risk calculator might involve addition of biomarker or genomic data and expansion of the study sample to include men from other populations, allowing general application.

\section{Conclusions}

A prostate cancer risk calculator (PCRC-MRI), which employs both clinical and MRI data, provides a useful tool to predict presence of csPCa at biopsy in North American men. Usage of PCRC-MRI would reduce unnecessary biopsies and requires only standard-of-care inputs. 


\section{References}

1. Norris JM, Kinnaird A, Margolis DJ, Padhani AR, Walz J, Kasivisvanathan V. Developments in MRI-targeted prostate biopsy. Curr Opin Urol. 2020;30(1):1-8.

2. Ahmed HU, Bosaily AE-S, Brown LC, et al. Diagnostic accuracy of multi-parametric MRI and TRUS biopsy in prostate cancer (PROMIS): a paired validating confirmatory study. Lancet. 2017;389(10071):815-822.

3. Kasivisvanathan V, Rannikko AS, Borghi M, et al. MRI-targeted or standard biopsy for prostate-cancer diagnosis. N Engl J Med. 2018;378(19):1767-1777.

4. Filson CP, Natarajan S, Margolis DJA, et al. Prostate cancer detection with magnetic resonance-ultrasound fusion biopsy: The role of systematic and targeted biopsies. Cancer. 2016;122(6):884-892.

5. Bjurlin MA, Carroll PR, Eggener S, et al. Update of the Standard Operating Procedure on the Use of Multiparametric Magnetic Resonance Imaging for the Diagnosis, Staging and Management of Prostate Cancer. J Urol. 2019:10-1097.

6. Liu W, Patil D, Howard DH, et al. Adoption of prebiopsy magnetic resonance imaging for men undergoing prostate biopsy in the United States. Urology. 2018;117:57-63.

7. Loeb S, Carter HB, Berndt SI, Ricker W, Schaeffer EM. Complications after prostate biopsy: data from SEER-Medicare. J Urol. 2011;186(5):1830-1834.

8. Halpern JA, Sedrakyan A, Dinerman B, Hsu W-C, Mao J, Hu JC. Indications, utilization and complications following prostate biopsy: New York state analysis. $J$ Urol. 2017;197(4):1020-1025.

9. Weiner AB, Manjunath A, Kirsh GM, et al. The Cost of Prostate Biopsies and their Complications: A Summary of Data on All Medicare Fee-for-Service Patients over 2 Years. Urol Pract. 2020;7(2):145-151.

10. Ankerst DP, Hoefler J, Bock S, et al. Prostate Cancer Prevention Trial risk calculator 2.0 for the prediction of low-vs high-grade prostate cancer. Urology. 2014;83(6):13621368 .

11. Van Den Bergh RCN, Roobol MJ, Wolters T, Van Leeuwen PJ, Schröder FH. The Prostate Cancer Prevention Trial and European Randomized Study of Screening for Prostate Cancer risk calculators indicating a positive prostate biopsy: a comparison. BJU Int. 2008;102(9):1068-1073.

12. Alberts AR, Roobol MJ, Verbeek JFM, et al. Prediction of high-grade prostate cancer following multiparametric magnetic resonance imaging: improving the Rotterdam European Randomized Study of Screening for Prostate Cancer Risk Calculators. Eur Urol. 2019;75(2):310-318.

13. Jones JS. Risk Calculators Are Only Applicable to Populations They Are Derived From. J Am Coll Cardiol. 2016;68(10):1148-1149.

14. Afshari Mirak* S, Bajgiran AM, Hosseiny M, et al. PD64-06 COMPARISON OF PERFORMANCE OF PI-RADSV2 AND A QUANTITIATIVE PI-RADSV1 BASED PROTOCOL IN 3T MULTIPARAMETRIC MRI FOR DETECTION, GRADING AND STAGING OF PROSTATE CANCER USING WHOLE MOUNT HISTOPATHOLOGY AS REFERENCE STANDARD IN 569 PATIENTS. $J$ Urol. 2019;201(Supplement 4):e1183-e1183.

15. Elkhoury FF, Felker ER, Kwan L, et al. Comparison of targeted vs systematic prostate 
biopsy in men who are biopsy naive: the Prospective Assessment of Image Registration in the Diagnosis of Prostate Cancer (PAIREDCAP) study. JAMA Surg. 2019.

16. Ahdoot M, Wilbur AR, Reese SE, et al. MRI-Targeted, Systematic, and Combined Biopsy for Prostate Cancer Diagnosis. N Engl J Med. 2020;382(10):917-928.

17. Mehralivand S, Shih JH, Rais-Bahrami S, et al. A magnetic resonance imaging-based prediction model for prostate biopsy risk stratification. JAMA Oncol. 2018;4(5):678685.

18. Vickers AJ, Elkin EB. Decision curve analysis: a novel method for evaluating prediction models. Med Decis Mak. 2006;26(6):565-574.

19. van Leeuwen PJ, Hayen A, Thompson JE, et al. A multiparametric magnetic resonance imaging-based risk model to determine the risk of significant prostate cancer prior to biopsy. BJU Int. 2017;120(6):774-781.

20. Siegel RL, Miller KD, Jemal A. Cancer statistics, 2020. CA Cancer J Clin. 2020;70(1):7-30.

21. Lovegrove CE, Miah S, El-Shater Bosaily A, et al. Comparison of Transrectal Ultrasound Biopsy to Transperineal Template Mapping Biopsies Stratified by Multiparametric Magnetic Resonance Imaging Score in the PROMIS Trial. $J$ Urol. 2019:10-1097.

22. Patel NU, Lind KE, Garg K, Crawford D, Werahera PN, Pokharel SS. Assessment of PI-RADS v2 categories $\geq 3$ for diagnosis of clinically significant prostate cancer. Abdom Radiol. 2019;44(2):705-712.

23. Distler FA, Radtke JP, Bonekamp D, et al. The value of PSA density in combination with PI-RADS ${ }^{\mathrm{TM}}$ for the accuracy of prostate cancer prediction. $J$ Urol. 2017;198(3):575-582.

24. Paller CJ, Wang L, Brawley OW. Racial Inequality in Prostate Cancer OutcomesSocioeconomics, Not Biology. JAMA Oncol. 2019;5(7):983-984.

25. Ballon J, Kinnaird A, Jayadevan R, et al. MRI-Guided Biopsy to Evaluate Prostate Cancer Severity in African-American Men. Urol Pract. 2019:10-1097.

26. Gross MD, Marks LS, Sonn GA, et al. Variation in Magnetic Resonance ImagingUltrasound Fusion Targeted Biopsy Outcomes in Asian-American Men: A Multicenter Study. J Urol. 2019:10-1097.

27. Marks LS, Kojima M, Demarzo A, et al. Prostate cancer in native Japanese and Japanese-American men: effects of dietary differences on prostatic tissue. Urology. 2004;64(4):765-771.

28. Rawla P. Epidemiology of prostate cancer. World J Oncol. 2019;10(2):63.

29. Ankerst DP, Boeck A, Freedland SJ, et al. Evaluating the PCPT risk calculator in ten international biopsy cohorts: results from the Prostate Biopsy Collaborative Group. World J Urol. 2012;30(2):181-187.

30. Püllen L, Radtke JP, Wiesenfarth M, et al. External validation of novel magnetic resonance imaging-based models for prostate cancer prediction. BJU Int. 2019.

\section{Figures and Tables}


Fig. 1. Flow diagram of participants. Of the final 2354, 1594 came from UCLA and 760 from Cornell. Study dates were between 9/2009 and 4/2019. GG: Gleason grade group; MRI: magnetic resonance imaging; MRGB; MRI-guided biopsy; PCa: prostate cancer.

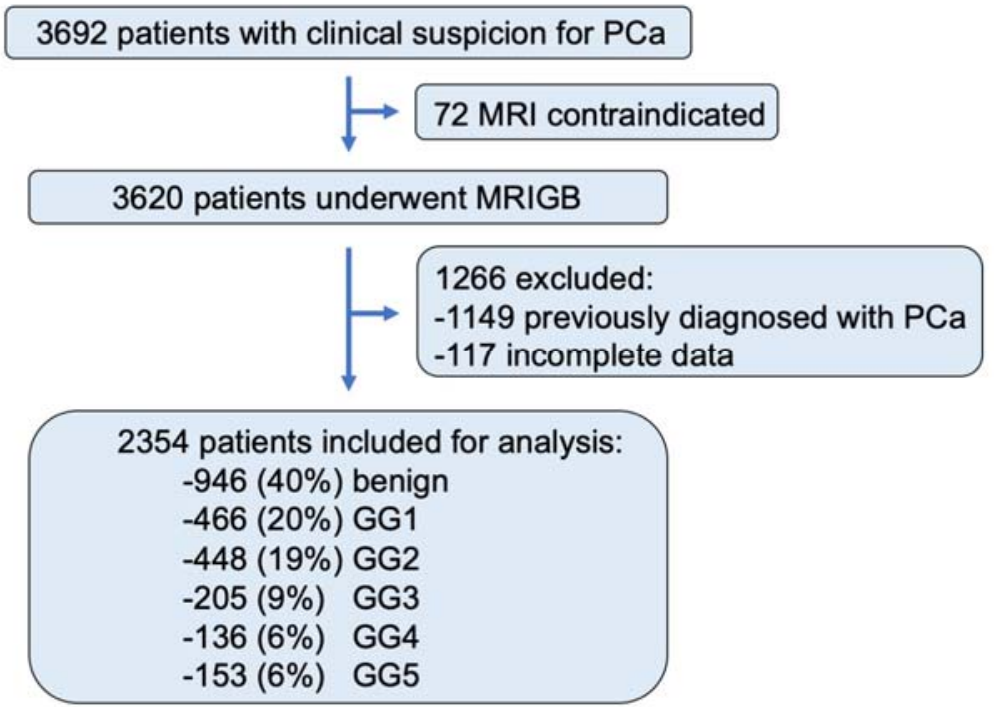

Fig. 2. Biopsy schema: magnetic resonance imaging (MRI)-targeted biopsies (green) and systematic biopsies (yellow), magnetic resonance imaging region of interest (MRI-ROI) (blue), and prostate (brown). Combined targeted and systematic biopsy was performed in all patients. ${ }^{15,16}$

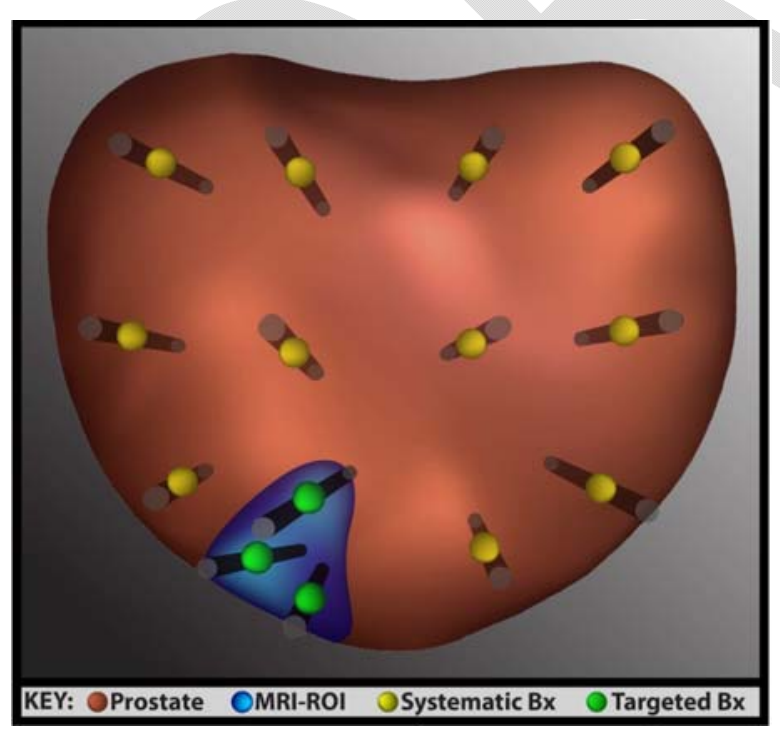

Fig. 3. ROC of models from the discovery cohort. (A) Overall comparison. Note that the clinical+MRI model is superior to clinical model (AUC 0.843 vs. 0.707, $p<0.0001$ ). (B) Componenets of the clinical+MRI model, compared to the full model. (C) Comparison of the 
clinical+MRI model to MRI+PSA density and MRI score alone. Clinical+MRI is superior to both MRI+PSA density (AUC 0.843 vs. $0.796, \mathrm{p}<0.0001$ ) and MRI alone models (AUC 0.843 vs. $0.760, p<0.0001)$. AUC: area under the curve; DRE: digital rectal exam; MRI: magnetic resonance imaging; PSA: prostate-specific antigen; ROC: receiver operating characteristic,

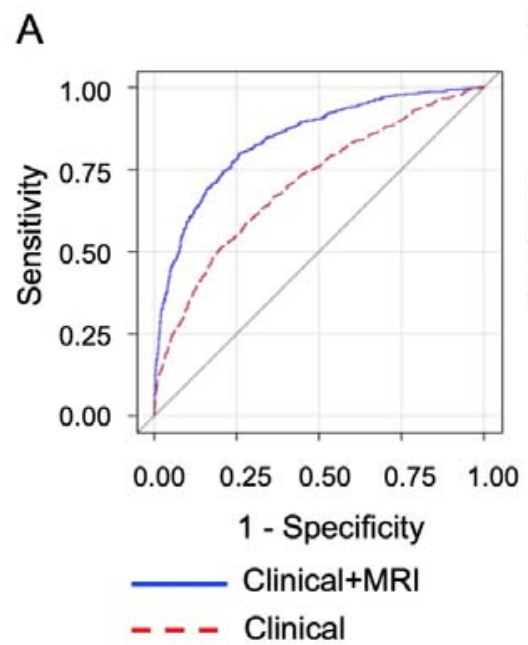

B

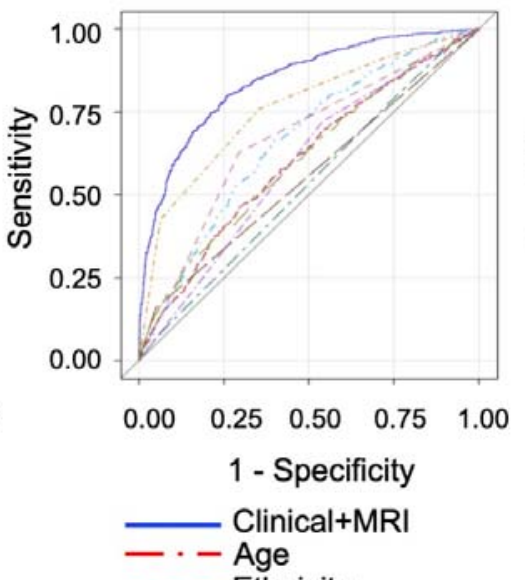

- - - Ethnicity

$-\cdots$ MRI score

- - Previous negative biopsy

- - Abnormal DRE

- - - PSA density

- - PSA

-..Prostate volume
C

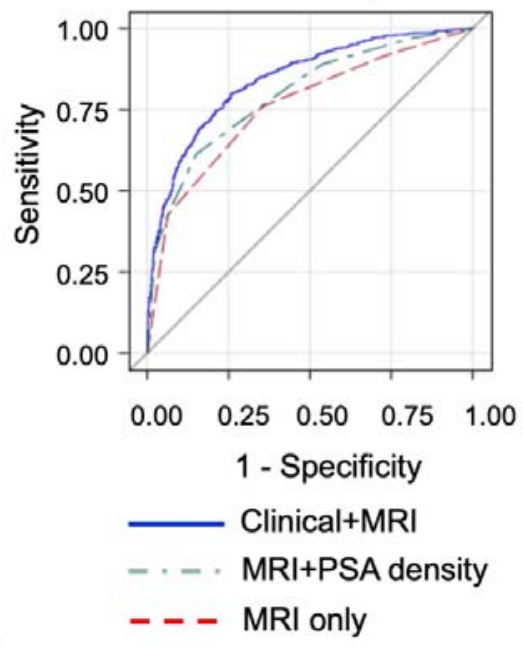

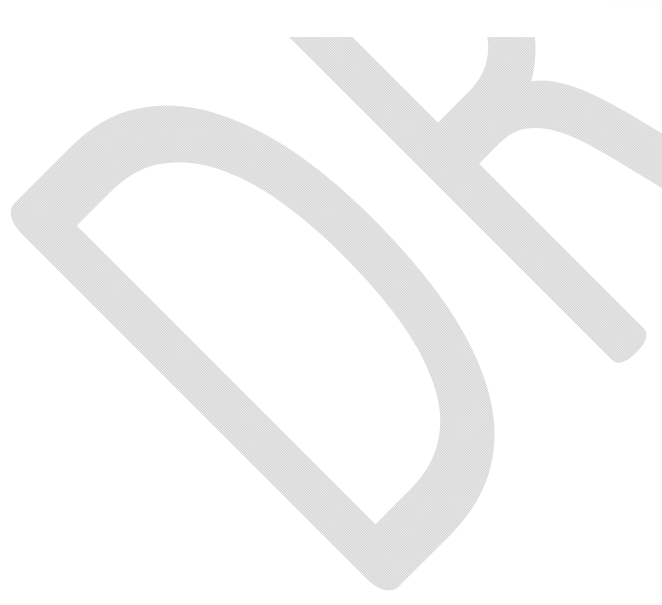




\begin{tabular}{|l|c|}
\hline \multicolumn{2}{|l|}{ Table 1. Baseline characteristics of study sample (n=2354) } \\
\hline Age (years), mean (SD) & $65.0(7.8)$ \\
\hline Ethnicity, n (\%) & $1491(63)$ \\
\hline Caucasian & $141(6)$ \\
\hline African American & $171(7)$ \\
\hline Asian & $116(5)$ \\
\hline Other & $435(18)$ \\
\hline Unknown & $231(10)$ \\
\hline Abnormal DRE, n (\%) & $1655(70)$ \\
\hline Yes & $468(20)$ \\
\hline No & $332(21)$ \\
\hline Unknown & $1220(79)$ \\
\hline Family history of prostate cancer ${ }^{*}, \mathrm{n}(\%)$ & \\
\hline Yes & $905(38)$ \\
\hline No & $1449(62)$ \\
\hline Previous negative biopsy, n (\%) & $6.5(4.7,9.5)$ \\
\hline Yes & $48(35,68)$ \\
\hline No & \\
\hline PSA (ng/mL), median (IQR) & $1344(57)$ \\
\hline Prostate volume (cc), median (IQR) & $1010(43)$ \\
\hline PSA density, n (\%) & \\
\hline$<0.15$ & $452(19)$ \\
\hline$\geq 0.15$ & $677(29)$ \\
\hline MRI score & $495(21)$ \\
\hline Negative ( $\leq 2)$ & \\
\hline 3 & \\
\hline 4 & \\
\hline 5 & \\
\hline
\end{tabular}

* $\mathrm{n}=1552$, UCLA data only. DRE: digital rectal exam; IQR: interquartile range; MRI: magnetic resonance imaging; PSA: prostate-specific antigen; SD: standard deviation. 


\begin{tabular}{|c|c|c|}
\hline & $\begin{array}{l}\text { Clinical model } \\
\text { OR }(95 \% \mathrm{CI})\end{array}$ & $\begin{array}{c}\text { Clinical + MRI model } \\
\text { OR (95\% CI) }\end{array}$ \\
\hline Age ay biopsy & $1.05(1.03,1.06)$ & $1.06(1.04,1.07)$ \\
\hline \multicolumn{3}{|l|}{ Ethnicity } \\
\hline African American vs. Caucasian & $1.25(0.82,1.92)$ & $1.27(0.78,2.07)$ \\
\hline Asian vs. Caucasian & $0.62(0.41,0.95)$ & $0.46(0.29,0.75)$ \\
\hline Other vs. Caucasian & $1.21(0.75,1.94)$ & $1.21(0.70,2.08)$ \\
\hline Unknown vs. Caucasian & $0.98(0.76,1.28)$ & $0.92(0.67,1.25)$ \\
\hline $\begin{array}{l}\text { DRE abnormality (yes vs. } \\
\text { no/unknown) }\end{array}$ & $3.06(2.15,4.34)$ & $2.37(1.56,3.63)$ \\
\hline Previous negative biopsy (yes vs. no) & $0.41(0.33,0.51)$ & $0.54(0.42,0.69)$ \\
\hline PSA & $1.06(1.04,1.08)$ & $1.02(1.00,1.04)$ \\
\hline PSA volume & & $0.98(0.98,0.99)$ \\
\hline PSA density & & $2.41(1.81,3.22)$ \\
\hline \multicolumn{3}{|l|}{ MRI score } \\
\hline 3 vs. negative & & $1.67(1.15,2.42)$ \\
\hline 4 vs. negative & & $3.96(2.79,5.62)$ \\
\hline 5 vs. negative & 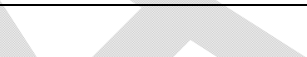 & $15.85(10.51,23.89)$ \\
\hline
\end{tabular}

CI: confidence interval; DRE: digital rectal exam; MRI: magnetic resonance imaging; PSA: prostate-specific antigen. 